\title{
Management of Dentigerous Cysts - A Review
}

\author{
Mihir Joshi ${ }^{1}$, Soumi Samuel², Abel Abraham³, Deeyah Miriam Deepak ${ }^{4}$ \\ 1,2,3 Department of Oral and Maxillofacial Surgery, A.B. Shetty Memorial Institute of \\ Dental Sciences (ABSMIDS), Nitte University, Deralakatte, Mangalore, Karnataka, \\ India. ${ }^{4}$ A.B. Shetty Memorial Institute of Dental Sciences (ABSMIDS), Nitte \\ University, Deralakatte, Mangalore, Karnataka, India
}

\section{ABSTRACT}

\section{BACKGROUND}

Lesions of a cystic nature have long been described in relation to the bones of the face. Over the course of the last four hundred years, such entities have been identified and studied in great detail; the evolution of systems of classification, the knowledge regarding the true nature of such phenomena, and the different ways of treating such maladies of the face have all undergone vast transformation since the 19th century. The introduction and development of anaesthetics, both general and regional, helped broaden the types of approaches to cysts of the orofacial region. Therapies of a conservative nature could be studied in greater detail with a larger population of patients, with the long term outcomes of such procedures yielding a significant amount of data, which in turn helped contrast such forms of management against more extensive and often radical procedures. Controversy has long surrounded the treatment of Dentigerous Cysts. Older techniques that had fallen out of favour amongst practicing surgeons returned decades later as serious contenders for becoming the treatment of choice for this particular pathology, the Partsch I technique being a prime example. The employment of the scientific method, conscientious effort towards ethics, and diligent recording of findings - whether prior to the surgical procedure or in the immediate and late post operative periods - all aided in the provision of a pool of knowledge that was made available to students and practitioners alike, providing clarity in the midst of opposing theories, guiding them in the selection of an appropriate treatment in response to cysts. Revolutions in medical imaging technology helped refine this process and will continue to serve the surgeon greatly in the pursuit of a treatment modality that is tailored to the needs of the patient and the demands of the lesion. Dentigerous cysts are anomalies that have been frequently encountered by practitioners, and the treatment for the same has long been discussed.

The purpose of this paper was to review the existing evidence in relation to the management of Dentigerous Cysts, and to highlight in particular the efficacy of decompression amongst paediatric patients. We conducted a narrative review of literature using electronic databases such as Pubmed, Scopus, and Google Scholar involving studies with data on the modes of management of Dentigerous Cysts. The list of included studies was reviewed to find more significant articles for relevant evidence.

\section{KEY WORDS}

Dentigerous Cyst, Enucleation, Marsupialization
Corresponding Author: Dr. Soumi Samuel,

Department of Oral and Maxillofacial Surgery, A.B. Shetty Memorial Institute of Dental Sciences (ABSMIDS), Nitte University, Deralakatte, Mangalore, Karnataka, India. E-mail: soumisamuel@gmail.com

DOI: $10.14260 /$ jemds/2021/400

How to Cite This Article: Joshi M, Samuel S, Abraham A, et al. Management of dentigerous cysts - a review. J Evolution Med Dent Sci 2021;10(26):1948-1953, 10.14260/jemds/2021/400

Submission 18-02-2021,

Peer Review 19-04-2021,

Acceptance 22-04-2021,

Published 28-06-2021.

Copyright (C) 2021 Mihir Joshi et al. This is an open access article distributed under Creative Commons Attribution License [Attribution 4.0 International (CC BY 4.0)] 


\section{BACKGROUND}

The concept of 'liquid tumours' was first put forth by a German surgeon by the name of Scultet in 1671. They were considered even back then to cause pressure within the jawbones that was significant enough to bring about some degree of expansion. "Dentigerous Cysts" (DC) as a term was first put forth in 1853 by James Paget; it was considered a broad term, encompassing all cysts that were thought to have their origin in dental tissue. It was only by 1976 , following attempts at classification, that a niche was created for the term, with Shear stating 'follicular' and 'dentigerous' cysts to be one and the same. ${ }^{2}$ In 1892, Carl Partsch proposed a 'cystotomy' as a means to halt expansion and address the negative effects of a build-up of pressure, thus giving birth to Marsupialisation. Kurt Thoma later developed this technique into 'Decompression', which - he stated - had the added benefits of allowing for pulp vitality to be maintained, lowering the risk of fracture to the maxilla or mandible, and preservation of important structures such as the maxillary antrum and the inferior alveolar nerve, showing that the risk of recurrence was acceptably low.

DCs have been studied over time in great detail, providing some insight into how they originate, the nature of their biology and the manifestations of the same in a general populace. Being a type of odontogenic cyst, the DC is a localized swelling of cystic nature associated with an impacted tooth commonly occurring in the mandible in association with the lower third molar, followed by the canine, third molar and central incisor of the maxillary region. ${ }^{3}$ It accounts for $20 \%$ of all jaw cysts with around $10 \%$ of impacted teeth manifesting this pathology. Usually an accidental finding on routine radiography, most DCs tend to present well into the second or third decades of life without overt prodromal symptoms. It is with the permanent dentition that the developmental DC is commonly associated, even more so with an impacted mandibular third molar.

In patients with DCs who are in the mixed dentition phase, the cause may be attributed to a deciduous tooth that loses vitality. It may cause bacteria or bacterial products to spread beyond the root apex, bringing about inflammation that can affect the follicle of the developing enamel organ. This is what is believed to initiate the build-up of fluid and eventual separation of the reduced enamel epithelium from the coronal portion of the tooth bud. In such patients, an asymptomatic presentation is rare, with the patient complaining of both pain and swelling. Such DCs are thought to be of an inflammatory origin, presenting early, that is within the first two decades of life, and are associated often with the developing lower premolar. This may be - in part - due to close proximity of the primary molar to the follicle of the growing permanent bicuspid.

Investigators for the most part seem partial to a developmental origin, while privy to the knowledge of the existence of DCs in the paediatric population. 4,5 Although there is a lack of clarity on the exact histogenesis of DCs, it is now known that the build-up of fluid between the crown and the reduced enamel epithelium, or between epithelial layers, causes the classic finding of this ailment. The intra-cystic fluid pressure for a dentigerous cyst as studied by Kubota et al. was found to be $258.2 \pm 160.9 \mathrm{~mm}$ of $\mathrm{Hg} / \mathrm{cm}^{2}$. Under a microscope, these cysts reveal to have a lining consisting of a fine layer of non-keratinized, stratified squamous epithelium. A cystic space is evident between the enamel and the epithelium, this being one of the criteria for diagnosis. According to a classification by Robinson in 1937, Dentigerous cysts are subdivided into simple, compound, eruption and heterotrophic categories. ${ }^{6}$

A routine panoramic radiographic examination can demonstrate a cyst accidentally which is indicative of a dentigerous cyst. The involved area may be asymptomatic clinically. Clinical presentation may be a swelling in the affected area. Patients may experience pain or show signs of inflammation if the dentigerous cysts are infected. These cysts may be associated with a supernumerary tooth, unerupted permanent teeth or an odontoma. Radiographic examination presents as a unilocular or pericoronal radiolucent lesion. However, this alone cannot be sufficient for a definite diagnosis of Dentigerous cyst because similar radiographic pictures are associated with other lesions of odontogenic variety such as keratocystic odontogenic tumour (odontogenic keratocyst), unicystic variety of ameloblastoma or adenomatoid odontogenic tumor. ${ }^{7}$ Microscopic examinations of a biopsy or surgically excised specimens present as a final diagnostic criterion for dentigerous cysts.

Treatment therapy for dentigerous cysts involve enucleation combined with extraction of the involved tooth. Initial marsupialization of larger cysts can be done in order to reduce the size of the bony defect prior to definitive enucleation. ${ }^{8}$ Epidemiological studies have shown a large occurrence of these cysts in children, therefore marsupialization can be performed to allow the eruption of the permanent tooth. The incomplete root development maintains the eruptive strength of the tooth aiding in its role to erupt. ${ }^{9}$ Knowledge that is continuing to expand to manage the parameters of care in pathological conditions of a dentigerous cyst is important. This review therefore intended to increase the understanding of the clinical features of this disease, its biologic nature and reaction to therapy helping clinicians to improve their skills and treatment outcomes.

MODALITIES FOR THE MANAGEMENT OF DENTIGEROUS CYSTS

Dentigerous Cyst Also Known as Follicular Cyst Classically solitary, it is only in conjunction with syndromes (for eg: Maroteaux - Lamy / cleidocranial dysplasia) that bilateral or multiple cysts are found. DC generally presents between the $2^{\text {nd }}$ and $3^{\text {rd }}$ decades of life, showing a male predilection wherein the ratio is $3: 2$ for male to female patients. Lee et al. in 2019 confirmed using the Wilcoxon Rank Sum Test that cysts were larger amongst the male population by a significant degree. 10

The typical follicular space is $3-4 \mathrm{~mm}$ and when the space is more than $5 \mathrm{~mm}$ a dentigerous cyst can be suspected. Transformation of the cyst into ameloblastoma, mucoepidermoid carcinoma or squamous cell carcinoma can also take place.

The radiographic presentation of dentigerous cysts appear as unilocular type $(89.1 \%)$ and multilocular (10.9\%) welldefined pericoronal radiolucencies centred on an impacted or unerupted tooth. ${ }^{11}$ 
Based on radiographic appearance, dentigerous cysts are classified into three types and are as follows: when radiolucency surrounds the crown of unerupted tooth it is classified as central type; lateral type - which encloses laterally along the tooth; and Circumferential type where the crown and root of the tooth are encircled 12,13

As per the American Association of Oral and Maxillofacial Surgeons (AAOMS) Guidelines outlined in 2017, the general management protocol for cysts are as explained below.

The pre-surgical assessment includes, a detailed history, a physical examination of clinical features, and radiographic presentation -

A. With the of help of aspiration and biopsy, definite diagnosis is achieved

B. Primary treatment

1. Observation, including clinical and serial radiographs evaluation (e.g., presumptive diagnosis of idiopathic bone cavity, traumatic bone cyst, unicameral bone cavity, Stafne cyst, and periapical radiolucency in the endodontically treated tooth)

2. Those with large cysts or unable to undergo enucleation or extirpation or for those in whom the potential damage to adjacent vital structures is high marsupialization and / or decompression for patients.

3. Not prone to recurrence - choice of treatment in enucleation.

4. In which complete removal by enucleation alone is known to be inadequate (curettage can be mechanical, physical, chemical, or a combination) enucleation and curettage for lesions.

5. For aggressive or recurrent cysts, the choice of surgical management is marginal or segmental resection. All biopsy samples must be submitted for histopathological examination.

Problems associated with Conventional Marsupialization are repeated occlusion of the stent by debris, necrosis and scarring of tissue adjacent to the stent. To counteract this problem the ideal marsupialization stent should have a design that prevents it from dislocating into or out of the bone cavity, it should be as small as not to interfere with daily mastication, easy to fix in soft tissue, daily irrigation of the cystic cavity should be possible, and importantly it should remain hygienic over the expected life of the device.

\section{Chemical Cautery}

Carnoy's solution is described by Cutler and Zollinger. The composition of Carnoy's solution is $1 \mathrm{~g}$ of ferric chloride [FeCl3] incorporated in Ethanol, chloroform and glacial acetic acid in a ratio of 6:3:1. In Modified Carnoy's solution, chloroform is removed and is currently accepted. The carnoy's solution chemical composition acts as a fixative agent that penetrates bone to a depth of $1.54 \mathrm{~mm}$ after 5 minutes of application. 14,15

\section{Cryotherapy / Cryosurgery}

Liquid nitrogen is used as a material of choice for the procedure, which boils at -196 C. After enucleation, the cystic cavity is treated with the liquid nitrogen. Freezing of the cavity walls is done until a frost forms, maintained for 1 minute with repeated sprays. Thawing should be slow; it causes natural cell death and osmotic imbalance. Freezing is then normally repeated at least 1 more time for completeness.

For cysts of less than $1.5 \mathrm{~cm}$, conducting jelly is used with a liquid nitrogen probe to freeze the whole mass. For larger cavities, liquid nitrogen must be sprayed into the cavity with the help of a long intravenous cannula. ${ }^{16}$

\section{Adjunctive Treatment}

1. Fixation is preferred to reduce the subject's potential for fracture and / or preserve function (eg, maxillomandibular bone plates)

2. Management of bone defects which are likely to persist or break down are done with-

a. haemostatic restorable sponge which is soaked in a solution containing an antibiotic or thrombin

b. autogenous cancellous bone

c. allografts

d. Rocky mountain graft

e. xenografts

f. secondary reconstruction for cases with potential for infection or recurrence, if primarily reconstructed, or those with systemic or local contraindications.

\section{Post Treatment Follow Up}

1. Radiograph in the primary l postoperative period

2. Aesthetic and functional assessment and follow up for recurrence

a. For non-recurrence - prone cysts (dentigerous) clinical and imaging examination until aesthetic form and / or functions are restored.

b. For recurrence - prone cysts (odontogenic keratocyst) clinical and imaging examination should be carried out for the patient's lifetime, annually for 5 years, then biannually if no recurrence

3. Patient should be informed to alert the treating doctor, if clinical signs or symptoms appear before regular scheduled follow - up appointment.

\section{Surgical Approach}

Complete Enucleation (Partsch II Technique)

For a developmental dentigerous cyst that presents in an adult patient in association with an impacted permanent tooth, the accept mode of surgical treatment is the complete enucleation of the pathology.

In addition, the removal of the associated impacted tooth is absolutely indicated. Such an aggressive form of therapy allows for total eradication of the DC while removing the risk of a recurrence by performing a surgical extraction on the tooth that was involved. It is a single-stage procedure with excellent long term results, showing a low rate of recurrence and high patient satisfaction.

Other advantages are as follows -

- The entirety of the pathological lining is made available for detailed histopathological study.

- Hospital stay is short, with ten days or less being sufficient for the purpose of postoperative care.

- The surgical cavity does not require packs that have to be regularly replaced. 
- Bone regeneration is spontaneous.

However, such intense and aggressive surgery is not advisable amongst paediatric patients who present with the inflammatory type of dentigerous cyst. The possible complications of the procedure itself, along with the risk of damaging important anatomic structures such as the maxillary sinus, the inferior alveolar nerve and associated vasculature are not outcomes that can scientifically or ethically be considered in a young patient. There also exists in literature an increased risk for fracture of the facial bones involved, with the mandible being the most common. The nature of the procedure also dictates the necessity to subject the patient to a general anaesthetic. In addition, such a procedure entails the removal of a tooth that would otherwise have been able to erupt according to the usual chronological schedule to take its place in the final dentition. This would preclude the possibility of a normal, balanced occlusion, leading to further problems in terms of mastication, stability of occlusal forces and their corresponding effect on the two temporomandibular joints.

\section{Marsupialisation (Partsch I Technique)}

It is therefore advisable to revert to the original modality of treatment - one that had fallen out of over the past few decades in opposition to the complete enucleation approach, only to rise again as the more preferable form of therapy in specific situations, such as amongst patients in the first or second decades of life-decompression.

The classical treatment modality of marsupialization or decompression are primarily the choice of treatment for inflammatory dentigerous cysts. This is recommended in order to preserve the tooth that is associated with the pathology. This also favours their eruption when it is difficult to reliably predict the tooth eruption.

Sometimes after marsupialization / decompression, eruption of the associated impacted teeth assumes an abnormal position. It is therefore highly advisable to use a multifaceted approach to the management of such patients. The oral pathologist, the oral and maxillofacial surgeon, and the orthodontist have a unique opportunity to coordinate their efforts to bring about a form of therapy that addresses each patient's specific problems. The identification of the anomaly, its precise surgical approach and the subsequent focus on preservation of the patient's dentition offers the opportunity to successfully remove the offending tissue, while helping the patient achieve a normal occlusion by employing traction of the impacted tooth into its final desired place. This is only made possible if adequate space exists, which again lends considerable weight to the argument of including the skills of an experienced orthodontist to aid in the initial planning stage of the treatment and also the final orthodontically activated traction. ${ }^{17}$

Marsupialization, also known as Decompression or Partsch I technique, is a surgical technique based on the incomplete removal of the lining of the pathological cavity, while focusing on reducing the effect the cyst has on the adjacent structures. The cyst shrinks gradually to become smaller. This procedure permits a break in the cystic wall and paves a way for the cystic wall to drain the contents of the cyst. The cyst wall is sutured to the oral mucosa. This procedure permits the eruption of the cyst associated tooth, with or without orthodontic traction.
The advantages of marsupialisation are:

- Smaller duration of surgical intervention

- Minimal trauma to the patient

- Adjacent anatomic structures such as the teeth, the floor of the nose, the inferior alveolar nerve and the maxillary sinus are left intact

The drawbacks of marsupialization are:

- Longer hospital stay

- Greater duration of postoperative care and follow up

- Requires a high degree of patient and parental compliance

- Subpar bony healing in patients older than 20 years of age

- The risk of recurrence is categorically stated to be higher than the Partsch II technique

- The lining of the dentigerous cyst if not removed in its entirety, can cause any ameloblastomas or carcinomas that may be developing in other parts of the lining and may be missed due to incomplete histological examination

- A secondary procedure may be necessary

- Repeated packing of the pathological cavity is required 18

\section{DISCUSSION}

The treatment for patients with a dentigerous cyst of an inflammatory - type that includes an impacted tooth should no longer be enucleation. Instead, a multi-disciplinary approach employing marsupialization along with orthodontic traction of the associated impacted tooth can be incorporated in the treatment plan. The traction of the impacted tooth performed by the orthodontist who is part of the team should be planned based on the availability of sufficient space for the impacted tooth to be brought into occlusion. Research has been carried out to assess the favourable period of time for planning the traction orthodontically in order to allow the encased tooth to erupt from the cyst.

These researchers concluded that the eruption of the tooth with a dentigerous cyst associated is predictable and may take place three months following marsupialization; in certain cases, a longer duration of traction maybe required. The factors that are in favour for the eruption of a cyst associated with tooth after marsupialization are disputable. Researchers reported that factors that influence eruption of tooth depends on the age, inclination and depth of tooth, formation of root, and availability of space. In contrast, other researchers concluded that the above mentioned factors were not remarkable and need not favour tooth eruption ${ }^{19}$

Nyimi et al. has concluded that an initial approach, that is a conservative surgical approach with primary closure of the subsequent defect (less than $4 \mathrm{~cm}$ ), reduces the morbidity of aggressive surgeries. It obtains the complete bone healing within twenty-four months of the postoperative period. The treatment of large cystic lesions when cases are carefully selected is marsupialization being most common. Evocyst is an attractive new approach for obtaining complete bone defect healing within 3 months. ${ }^{20}$

Kimura et al. have developed a normal method for the treatment of odontogenic cysts. They have developed a silicone based tube as a decompression device. ${ }^{21}$ Núñez et al. has described an evacuator for odontogenic cysts (Evocyst), which is a closed and active (vacuum like) drain system. This is to promote osteogenesis and also to manage odontogenic 
cysts by means of active intra-cystic negative pressure. This device reveals a high rate of a new bone formation. Using the new device, a new bone formation takes place within less than three months, compared to conventional decompression that employs passive drains which tend to require up to twelve months to heal.22

Johnson et al. in 1994 reported the risk that occurs when considerable amounts of odontogenic epithelium remain within the affected bony cavity for a long duration. They suggested that the epithelium that positions around the crown of an impacted tooth may not necessarily undergo an immediate transformation into a dentigerous cyst. They also reported that the average age of occurrence of squamous carcinoma arising in a dentigerous cyst is 60.8 years. The pluripotent nature of the odontogenic epithelium around the crown of tooth determines its increased chance to become cystic or malignant in the future. ${ }^{23}$

Literature reports of a patient who at 16 months of age is the youngest patient ever reported with a carcinoma arising in a dentigerous cyst. This case satisfies all the existing criteria for this rare lesion. It projects that it is distinct on the basis of age of onset and the absence of a chronic lymphocytic infiltrate. The advanced age of previously reported cases of squamous cell carcinoma arising in a dentigerous cyst and the associated chronic inflammatory changes seen histologically have favoured a speculation that these carcinomas occur after a slow malignant change of the odontogenic epithelium. $4,24,25$

\section{CONCLUSIONS}

The Partsch I technique is one of the oldest known treatment modalities developed to combat dentigerous cysts. It has experienced periods of regression and resurgence amongst practitioners in terms of favourability. Now, however, it is clear that, provided the option is presented to the right patient of the right age with a high compliance, Marsupialisation offers a more conservative surgical approach to the treatment of this pathology, addressing both the concerns of the increase in pressure within the bone that the cyst causes, while also allowing for the retention of the involved tooth and its eventual repositioning into a desirable location as an erupted tooth in the oral cavity.

The immediate eruption of the involved tooth encased within the cyst can be carried out by Marsupialization therapy. However, marsupialization does not favour spontaneous eruption of the impacted tooth, and in certain cases the impacted tooth needs to be sacrificed. This might cause a deficiency of bone in the alveolar region. Establishing the occlusion in dentigerous cysts of the jaw presents a tailored multidisciplinary approach that includes rehabilitation.

Financial or other competing interests: None.

Disclosure forms provided by the authors are available with the full text of this article at jemds.com.

\section{REFERENCES}

[1] Sivapathasundharam B. Shafer's Textbook of Oral Pathology. India: Elsevier Health Sciences 2020 Jul 15.
[2] Castro-Núñez J. Decompression of odontogenic cystic lesions: past, present and future. J Oral Maxillofac Surg 2016;74(1):104.e1-9.

[3] Deboni MCZ, Brozoski MA, Traina AA, et al. Surgical management of dentigerous cyst and keratocystic odontogenic tumor in children: a conservative approach and 7-year follow-up. J Appl Oral Sci 2012;20(2):282-5.

[4] Arce K, Streff CS, Ettinger KS. Pediatric odontogenic cysts of the jaws. Oral Maxillofac Surg Clin 2016;28(1):21-30.

[5] Azaz B, Shteyer A. Dentigerous cyst associated with second mandibular bicuspids in children: report of five cases. ASDC J Dent Child 1973;40(1):29-31.

[6] Robinson HBG. Classification of cysts of the jaws. Am J Ortho Oral Surg 1945;31(6):370-5.

[7] Regezi JA, Sciubba JJ, Jordan RC. Oral pathology: clinical pathologic correlation. $6^{\text {th }}$ edn. St. Louis: Saunders Elsevier 2012:246-69.

[8] Pinheiro RDS, Castro GF, Roter M, et al. An unusual dentigerous cyst in a young child. Gen Dent 2013;61(2):62-4.

[9] Picciotti M, Divece L, Parrini S, et al. Replantation of tooth involved in dentigerous cyst: a case report. Eur J Paediatr Dent 2012;13(4):349-51.

[10] Lee H, Lee SJ, Seo BM. Investigation of postoperative complications of intrabony cystic lesions in the oral and maxillofacial region. J Oral Maxillofac Surg 2019;77(9):1823-31.

[11] Terauchi M, Akiya S, Kumagai J, et al. An analysis of dentigerous cysts developed around a mandibular third molar by panoramic radiographs. Dent J (Basel) 2019;7(1):13.

[12] Shear M, Speight P. Cysts of the oral and maxillofacial regions. $4^{\text {th }}$ edn. Munksgaard, Oxford: Blackwell 2007:6970.

[13] Rajendran R. Shafer's textbook of oral pathology. India: Elsevier 2009.

[14] Pogrel MA. The keratocystic odontogenic tumor. Oral Maxillofac Surg Clin North Am 2013;25(1):21-30.

[15] Abdullah WA. Surgical treatment of keratocystic odontogenic tumour: a review article. Saudi Dent J 2011;23(2):61-5.

[16] Lieblich SE, Kleiman MA, Mj JZ. Parameters of care: clinical practice. Guidelines for oral and maxillofacial surgery. (AAOMS ParCare 2012). Journal of Oral and Maxillofacial Surgery 2012;70(e50).

[17] Aoki N, Ise K, Inoue A, et al. Multidisciplinary approach for treatment of a dentigerous cyst-marsupialization, orthodontic treatment and implant placement: a case report. J Med Case Rep 2018;12(1):1-7.

[18] Bodner L. Cystic lesions of the jaws in children. Int J Pediatr Otorhinolaryngol 2002;62(1):25-9.

[19] Nyimi BF, Yifang Z, Liu B. The Changing landscape in treatment of cystic lesions of the jaws. J Int Soc Prev Community Dent 2019;9(4):328-37.

[20] Kimura M, Ishibashi K, Shibata A, et al. A new decompression device for treating odontogenic cysts using a silicone tube. Br J Oral Maxillofac Surg 2020;58(1):116-7.

[21] Castro-Núñez J, Rey D, Amaya L. An innovative intracystic negative pressure system to treat odontogenic cysts. J Craniofac Surg 2017;28(7):1883-4. 
[22] Gulbranson SH, Wolfrey JD, Raines JM, et al. Squamous cell carcinoma arising in a dentigerous cyst in a 16-month-old girl. Otolaryngol Head Neck Surg 2002;127(5):463-4.

[23] Manganaro AM, Cross SE, Startzell JM. Carcinoma arising in a dentigerous cyst with neck metastasis. Head Neck 1997;19(5):436-9.
[24] Sharma M, Pardhe ND, Gupta N, et al. Inflammatory dentigerous cyst: a rare entity. J Res Adv Dent 2014;3(Suppl 2):4-7.

[25] Johnson LM, Sapp JP, McIntire DN. Squamous cell carcinoma arising in a dentigerous cyst. J Oral Maxillofac Surg 1994;52(9):987-90. 